\title{
A Steric Block in Translation Caused by the Antibiotic Spectinomycin
}

\author{
Maria A. Borovinskaya ${ }^{\# \dagger}$, Shinichiro Shoji ${ }^{\# \ddagger}$, James M. Holton ${ }^{\dagger, \uparrow}$, Kurt Fredrick ${ }^{\ddagger, \S}$, and \\ Jamie H. D. Cate ${ }^{\dagger, \|,{ }^{* *}, *}$ \\ †Physical Biosciences Division, Lawrence Berkeley National Laboratory, Berkeley, California \\ 94720 \\ ¥Department of Microbiology, The Ohio State University, Columbus, Ohio 43210 \\ §Ohio State Biochemistry Program, The Ohio State University, Columbus, Ohio 43210 \\ IDepartment of Biochemistry and Biophysics, University of California, San Francisco, California \\ 94158 \\ "Department of Chemistry, University of California, Berkeley, California 94720 \\ ${ }^{*}$ Department of Molecular and Cell Biology, University of California, Berkeley, California 94720 \\ \# These authors contributed equally to this work.
}

\begin{abstract}
The widely used antibiotic spectinomycin inhibits bacterial protein synthesis by blocking translocation of messenger RNA and transfer RNAs on the ribosome. Here, we show that in crystals of the Escherichia coli 70S ribosome spectinomycin binding traps a distinct swiveling state of the head domain of the small ribosomal subunit. Spectinomycin also alters the rate and completeness of reverse translocation in vitro. These structural and biochemical data indicate that in solution spectinomycin sterically blocks swiveling of the head domain of the small ribosomal subunit and thereby disrupts the translocation cycle.
\end{abstract}

Spectinomycin, a broad-spectrum antibiotic that selectively targets bacterial ribosomes (13 ), remains important in clinical and veterinary use, as it induces only low levels of bacterial resistance (4-6). Despite decades of worldwide use of the drug, the molecular mechanism by which spectinomycin inhibits translation remains unclear.

Spectinomycin, an aminocyclitol constituted from two glucose moieties (7) (Figure 1, panel a), inhibits translocation of transfer RNAs (tRNAs) and messenger RNA (mRNA) on the ribosome (8-10), the step that follows the formation of each peptide bond. Translocation, catalyzed by the GTPase elongation factor G (EF-G), involves multiple large conformational

*Corresponding author, jcate@lbl.gov..

Supporting Information Available: This material is available free of charge via the Internet.

Accession Codes: The coordinates and structure factors have been deposited in the Protein Data Bank with accession codes 2QOU, 2QOV, 2QOW, and 2QOX (70S ribosome in complex with spectinomycin), and 2QOY, 2QOZ, 2QP0, and 2QP1 (70S ribosome in complex with spectinomycin and neomycin). 
changes in the ribosome $(9,11-15)$. Translocation is thought to begin with a ratchet-like motion of the small (30S) ribosomal subunit relative to the large (50S) ribosomal subunit $(11,15,16)$, followed by swiveling of the head of the small subunit $(12,13)$ and "unlocking" (opening) of the tRNA binding groove to allow peptidyl (P-site) tRNA to pass into the Exit $(\mathrm{E})$ site $(13,14,17,18)$. These conformational changes lead to discrete movements of bound tRNAs, where the tRNAs move first with respect to the 50S subunit into hybrid binding states and then move with respect to the 30S subunit (19). Unlike most of the translocation inhibitors, spectinomycin slows but does not completely abolish multiple-turnover translocation (9). It also does not induce mRNA miscoding $(20,21)$.

The binding site for spectinomycin, in RNA helix 34 (h34) of the head domain of the 30S subunit, resides near the single ribosomal RNA (rRNA) helix that connects the head domain to the rest of the small subunit (i.e., neck of the 30S subunit) and faces the mRNA binding groove (22-26) (Figure 1, panels b and c; Supplementary Figure 1). Biochemical data (9, 24, $25,27)$ and the structure of spectinomycin bound to the 30 S ribosomal subunit (26) suggested that spectinomycin might inhibit translocation by causing or preventing conformational changes in the ribosome that potentially involve $h 34$ or the head of the $30 \mathrm{~S}$ subunit. However, since translocation depends on coordinated movements within the intact $70 \mathrm{~S}$ ribosome (15), the explanation for how spectinomycin inhibits translocation has not been established. To shed light on the mechanism by which spectinomycin inhibits translocation, we conducted structural and biochemical studies of the intact Escherichia coli $70 \mathrm{~S}$ ribosome in complexes with spectinomycin or with both spectinomycin and the aminoglycoside neomycin (Figure 1, panel a).

\section{RESULTS AND DISCUSSION}

The crystals of the E. coli 70S ribosome that were used for antibiotic soaks in the present experiments contain two molecules of the ribosome per asymmetric unit, termed ribosomes I and II (13). The small subunits of ribosomes I and II adopt remarkably different conformations, with the head of the 30S subunit swiveled around the neck helix in ribosome II, when compared to I, by $6^{\circ}$ in a trajectory that follows the path of tRNAs through the ribosome during translocation (Supplementary Figure 2).

Strikingly, binding of spectinomycin to the ribosomes within the crystals induced a global conformational change of the head domain of the small ribosomal subunit in ribosome II, the ribosome further along the tRNA trajectory from the P site towards the E site (Figure 2; Supplementary Figure 3) (13), whereas the conformation of ribosome I did not change. Spectinomycin binding to h34 near the neck helix of the 30S subunit caused the $30 \mathrm{~S}$ head domain of ribosome II to rotate around the neck back to a conformation closer to that of ribosome I, that is, closer to a pretranslocation or less swiveled conformation (13).

The ability of spectinomycin to trap the entire head of the small ribosomal subunit (molecular mass of $\sim 300 \mathrm{kDa}$ ) in a less swiveled conformation within crystals, clearly seen in the difference Fourier electron density maps (Figure 2, panels a and b), came as a surprise. This result indicates that in solution spectinomycin binding might sterically block movement of the head of the small subunit into the fully swiveled state of ribosome II and 
stabilize a conformation of the small subunit more like that of ribosome I. Within the crystals, the head domain of ribosome II rotated $\sim 1-2^{\circ}$ upon binding of spectinomycin (Figure 2, panels c and d; Supplementary Figure 3), with this effect likely to be amplified in the absence of crystal contacts.

In the present structures, spectinomycin possesses a single binding site on the ribosome, in the stem of h34 of the small ribosomal subunit (Figure 1, panels b and c; Supplementary Figure 1). The conformation of spectinomycin and its interactions with rRNA are similar to those observed in the structure of the 30S ribosomal subunit complexed with spectinomycin (26) (Figure 1, panel c; Supplementary Figure 1). Spectinomycin binds in the minor groove of h34 and contacts nucleotides G1064, C1066, G1068, C1192, and G1193, in agreement with chemical protection data (22) and mutagenesis studies $(23,24)$. Residue Lys 25 of ribosomal protein S5 also approaches spectinomycin (Figure 1, panel c; Supplementary Figure 1) and could potentially contact the drug in certain conformations of the $30 \mathrm{~S}$ subunit.

Neomycin possesses two adjacent binding sites on the 70S ribosome, one in $\mathrm{h} 44$ of the small subunit and the other in H69 of the large subunit (28) (Figure 1, panel b). These binding sites are located in the highly dynamic intersubunit region, next to the aminoacyl (A) and peptidyl (P) tRNA binding sites (28). The structure of the 70S ribosome with both spectinomycin and neomycin bound revealed that binding of neomycin was essentially identical to that observed previously in the complex of the 70S ribosome with neomycin alone (28), indicating that binding of the aminoglycoside and spectinomycin are structurally independent of each other. Notably, neomycin binding had no appreciable effect on the ability of spectinomycin to bind to ribosome II and trap the less swiveled conformation of the small subunit head (Figure 2). These data indicate that the two antibiotics target different steps of the translocation reaction on the ribosome.

Recent experiments revealed that several antibiotics that inhibit forward translocation are able to facilitate reverse translocation of mRNA and tRNAs on the ribosome even in the presence of EF-G and GTP (29). In contrast to neomycin and other translocation inhibitors (29), spectinomycin showed little effect on reverse translocation in the presence of EF-G and GTP but significantly inhibited the ability of neomycin to favor the pretranslocation (PRE) state in this assay (Figure 3, panels a and b). We also probed the effects of spectinomycin and neomycin on the spontaneous reverse translocation reaction, that is, in the absence of EF-G and GTP (Figure 3, panels a and c; Table 1; Supplementary Figure 4) $(29,30)$. Whereas neomycin increased the rate of spontaneous reverse translocation without affecting the final populations of post-translocation (POST) and PRE complexes, spectinomycin decreased both the rate and extent of spontaneous and neomycin-promoted reverse translocation (Figure 3, panel c; Table 1; Supplementary Figure 4).

The above structural and biochemical data suggest that spectinomycin specifically targets a transient intermediate in translocation. As noted earlier, translocation likely proceeds through a series of large conformational changes in the whole ribosome, beginning with a ratchet-like motion of the $30 \mathrm{~S}$ subunit relative to the $50 \mathrm{~S}$ subunit $(11,15,16)$. The ratcheting motion would not be sufficient for translocation (13), and a series of additional rearrangements would contribute to completing translocation, including disengagement of 
the minor-groove readout of the A-site tRNA (31), swiveling of the head domain of the small subunit $(12,13)$, and finally, opening of the tRNA binding groove to allow P-site tRNA to enter the E site $(13,14,17,18)$. The present structural results indicate that spectinomycin likely inhibits translocation by specifically blocking the swiveling of the head of the small subunit, consistent with recent kinetic experiments (18). Spectinomycin should allow ratcheting of the two ribosomal subunits to occur but would then freeze the head of the small subunit in a conformation close to that in ribosome I, whereas a fully rotated head, as in ribosome II, coupled with opening of the tRNA binding groove would be required to complete translocation $(12,13)$. Neomycin, in contrast, is thought to target the pretranslocation state of the ribosome, either prior to or following the formation of the ratcheted state (28). Whereas spectinomycin has little or no effect on peptidyl-tRNA affinity for the A site (9) but impacts 30S head dynamics (Figure 2; Supplementary Figure 3), aminoglycosides stabilize peptidyl-tRNA in the A site (9). Consistent with their differing effects on translocation (9) and reverse translocation reactions (Figure 3; Supplementary Figure 4), spectinomycin and neomycin target different sites on the ribosome (Figure 1, panel b) (28) and do not interfere with mutual binding.

In the presence of spectinomycin, either with or without neomycin, the spontaneous reverse translocation reactions followed a double exponential equation, that is, they were governed by two competing processes (Figure 3, panel c; Table 1; Supplementary Figure 4;

Supplementary Information). A plausible explanation for this observation is that there is a competition between binding of spectinomycin and either binding of E-site tRNA, which initiates reverse translocation, or opening of the tRNA binding groove between the $\mathrm{P}$ and $\mathrm{E}$ sites on the 30S subunit (13). Although we cannot distinguish at present whether spectinomycin inhibition precedes E-site tRNA binding or opening of the tRNA binding groove, the present data indicate that inhibition of head swiveling by spectinomycin occurs before the rate-limiting step of the spontaneous reverse translocation reaction. Notably, Peske et al. (9) observed biphasic kinetics of forward translocation in the presence of EF-G and spectinomycin, with higher concentrations of spectinomycin favoring the accumulation of more slowly translocating ribosome complexes. The fact that we observe a similar effect in the absence of EF-G in the reverse translocation reaction supports the notion that the ribosome itself, independent of the presence of EF-G, exists in two conformations that have different intrinsic abilities to translocate or reverse translocate tRNAs. By microscopic reversibility, spectinomycin likely affects $30 \mathrm{~S}$ head swiveling in the same way in both forward and reverse translocation reactions.

The conformational change caused by spectinomycin binding may additionally inhibit translocation by affecting the mRNA unwinding and movement on the ribosome (Figure 4). The average diameter of the mRNA downstream tunnel encircled by proteins $\mathrm{S} 3, \mathrm{~S} 4$, and S5, where mRNA unwinding takes place, is $\sim 15 \AA$ in the pretranslocation state, barely enough for the mRNA to fit (32) (Figure 4). Rotation of the head domain of the 30S subunit, which includes protein S3, occurs along the path of mRNA entering the A site (33) and results in a shearing movement of protein $\mathrm{S} 3$ in the head domain relative to proteins $\mathrm{S} 4$ and $\mathrm{S} 5$ in the 30S subunit shoulder. This shearing movement changes the shape and dimensions of the mRNA tunnel and likely contributes to mRNA unwinding by proteins S3, S4, and S5 
(32). Freezing of the head domain of the small subunit upon spectinomycin binding may therefore reduce the intrinsic helicase activity of the ribosome (32). The ability of spectinomycin to restrict rotation of the $30 \mathrm{~S}$ subunit head domain could also impede the forward motion of mRNA and therefore translocation.

Our study reveals the mechanism by which spectinomycin may inhibit tRNA and mRNA translocation on the ribosome and provides new insights into the sequence of structural rearrangements in the ribosome that are required for translocation. Similar structural changes may also be critical for the process of mRNA unwinding, involving a shearing of the $30 \mathrm{~S}$ subunit head relative to the $30 \mathrm{~S}$ subunit body. These results demonstrate the necessity of probing aspects of ribosomal translocation using the intact $70 \mathrm{~S}$ ribosome.

\section{METHODS}

\section{Crystallization, Data Collection, and Structure Refinement}

Ribosomes from E. coli strain MRE600 depleted of protein S1 were crystallized as described previously (13). Large single crystals were cryoprotected with buffers containing 20\% (v/v) 2-methyl-2,4-pentanediol (MPD), 3\% (w/v) PEG 8000, 24.1\% (v/v) PEG 400, 35 $\mathrm{mM} \mathrm{MgCl}_{2}, 350 \mathrm{mM} \mathrm{NH}_{4} \mathrm{Cl}, 1 \mathrm{mM}$ spermine, $0.5 \mathrm{mM}$ spermidine, and $60 \mathrm{mM}$ HEPES, $\mathrm{pH}$ $=7.0$, prior to flash-freezing in liquid nitrogen. The antibiotics at saturating concentrations, $0.1 \mathrm{mM}$ spectinomycin (Sigma) and $0.01 \mathrm{mM}$ neomycin (a mixture of neomycin $\mathrm{B}$ and $\mathrm{C}$, Sigma) $(8,22,34-36)$, were soaked into crystals for $24 \mathrm{~h}$ during cryostabilization.

Diffraction data for each complex were measured from multiple crystals (Table 2) cooled to $93 \mathrm{~K}$ using $0.1-0.3^{\circ}$ oscillations at the SIBYLS (12.3.1) beamline at the Advanced Light Source, which is equipped with an ADSC Q315 area detector. A modified strategy algorithm was used to optimize data measurement from multiple crystals. Data were reduced and scaled using Denzo/Scalepack (37) and Truncate (38) (Table 2). The crystals diffract X-rays anisotropically, as indicated by the completeness of the datasets as a function of resolution in Table 2 and Supplementary Figure 5.

The $3.5 \AA$ structure of the $E$. coli 70 S ribosome (13) was used as the starting model for further refinement in CNS (39). The model was first subjected to rigid body refinement against the antibiotic-bound data. The initial models of spectinomycin (40) and neomycin were manually docked into difference Fourier electron density maps, with apo-70S ribosomes as the reference and phases derived from Pirate density modification (41). The topology and parameter files describing each antibiotic were generated with HIC-Up (40). The models were then refined using rounds of manual rebuilding with $\mathrm{O}$ (42) and torsional dynamics (39). The refinement statistics are presented in Table 2. Following torsional dynamics refinement, electron density maps were generated using Pirate-derived phases (41).

\section{Toeprinting Assays}

The position of mRNA on the ribosome in translocation and reverse translocation experiments was determined by toeprinting, essentially as described previously (29) (Figure 3, panel a). All experiments were carried out in polymix buffer (43). Briefly, mRNA 292 
(Supplementary Figure 6) $(0.5 \mu \mathrm{M})$ annealed to a [ $\left.{ }^{32} \mathrm{P}\right]$-labeled primer was added to ribosomes depleted of protein $\mathrm{S} 1(0.7 \mu \mathrm{M})$, along with deacylated tRNA ${ }^{\mathrm{fMet}}(1 \mu \mathrm{M})$, and incubated at $37^{\circ} \mathrm{C}$ for $20 \mathrm{~min}$ to allow tRNA ${ }^{\mathrm{fMet}}$ to bind the ribosomal P site. The PRE complex was then formed by adding $N$-acetyl-Val-tRNA ${ }^{\mathrm{Val}}$ (to $1 \mu \mathrm{M}$ ) and incubating at 37 ${ }^{\circ} \mathrm{C}$ for 10 min to allow this tRNA to bind the ribosomal A site. The resulting complex was then diluted by $20 \%$ into polymix buffer containing GTP $(0.3 \mathrm{mM})$ and EF-G $(1 \mu \mathrm{M})$ and incubated at $37^{\circ} \mathrm{C}$ for $10 \mathrm{~min}$ to form a POST complex. Finally, the POST complex was diluted by $10 \%$ in the presence of either water or saturating concentrations of spectinomycin $(0.1 \mathrm{mM})$, neomycin $(0.1 \mathrm{mM})$, or both neomycin and spectinomycin $(0.1 \mathrm{mM}$ of each) and incubated for $10 \mathrm{~min}$ at $37^{\circ} \mathrm{C}$. Aliquots were removed for primer extension analysis at each stage of complex formation.

For primer extension reactions, each aliquot above was treated with AMV reverse transcriptase, and extension products were resolved by $6 \%(\mathrm{v} / \mathrm{v})$ polyacrylamide denaturing gel electrophoresis, as described previously (29). Toeprints were quantified using a phosphorimager (Molecular Dynamics) as follows. The percentage of POST complex was calculated as \% POST $=100 \times($ post $) /($ pre + post $)$, where pre and post are intensities of toeprints corresponding to PRE and POST complexes, respectively. The PRE complex corresponds to the sum of toeprints at positions +16 and +17 on the mRNA, whereas the POST complex corresponds to the toeprint at +19 on the mRNA (44).

To measure the rate of spontaneous reverse translocation (29), the P-site binding complex was formed as described above, using $N$-acetyl-Val-tRNA ${ }^{\mathrm{Val}}(1 \mu \mathrm{M})$. Reverse translocation was initiated by adding deacylated $\operatorname{tRNA}^{\mathrm{fMet}}(8 \mu \mathrm{M})$ to bind the E site at time $t=0$, and the reaction was incubated at $37^{\circ} \mathrm{C}$. The antibiotics neomycin $(0.1 \mathrm{mM})$, spectinomycin $(0.1$ $\mathrm{mM})$, or both neomycin and spectinomycin $(0.1 \mathrm{mM}$ of each) were also added at time $t=0$. A 2- $\mu \mathrm{L}$ aliquot was removed at each time point, immediately added to $10 \mu \mathrm{L}$ of prewarmed extension mix, and further incubated at $37{ }^{\circ} \mathrm{C}$ for $2 \mathrm{~min}$. The extension products were resolved by $6 \%(\mathrm{v} / \mathrm{v})$ polyacrylamide denaturing gel electrophoresis as described above. The fraction of POST complex at time $t, F(t)$, was determined by calculating $F(t)=[($ post $) /($ pre + post) $] / F_{\mathrm{P}}$, where $F_{\mathrm{P}}$ is the equivalent value derived from the $\mathrm{P}$ lane. The data were then plotted as a function of time and fit to either single or double exponential functions: $F(t)=$ $\left.F_{0}+A_{1} \times \exp \left(-k_{\mathrm{obs} 1} \times t\right)\right]$ or $\left.F(t)=F_{0}+A_{1} \times \exp \left(-k_{\mathrm{obs} 1} \times t\right)+A_{2} \times \exp \left(-k_{\mathrm{obs} 2} \times t\right)\right]$, where $A_{1}$ and $A_{2}$ are the amplitudes, and $k_{\mathrm{obs} 1}$ and $k_{\mathrm{obs} 2}$ are the observed rate constants (Table 1) (29). The single versus double type of exponential fit was deciphered on the basis of the residuals, i.e.. the differences in observed and calculated values, plotted for each type of a fit (Supplementary Figure 4). In the control experiment (water) or in the presence of neomycin, the data fit well to the single exponential function (Figure 3, panel c; Table 1;

Supplementary Figure 4). In the presence of spectinomycin, both with and without neomycin added, the reverse translocation time courses could only be fit to a double exponential function (Figure 3, panel c; Table 1; Supplementary Figure 4; Supplementary Information).

In the spontaneous reverse translocation experiments with spectinomycin preincubation, 0.1 $\mathrm{mM}$ of spectinomycin was incubated with the reaction mixture for $5 \mathrm{~min}$ at $37^{\circ} \mathrm{C}$ prior to adding deacylated tRNA ${ }^{\mathrm{fMet}}(8 \mu \mathrm{M})$ and either $0.1 \mathrm{mM}$ of neomycin or water at time $t=0$. 
The reactions and analyses were carried out as described above. The data fit to a double exponential function (Figure 3, panel c; Table 1).

\section{Figure Preparation}

The figures were made using programs Ribbons (45), ISIS Draw (MDL Information Systems, Inc.), Origin (OriginLab), and Adobe Photoshop (Adobe Systems, Inc.).

\section{Supplementary Material}

Refer to Web version on PubMed Central for supplementary material.

\section{Acknowledgment}

We thank K. Frankel, S. Classen, and G. Meigs for help with data measurement at the SIBYLS and 8.3.1 beamlines at the Advanced Light Source; W. Wintermeyer and R. Hille for helpful discussions; A. Borovinskiy for help with computational data handling, figure preparation, and overall support; and J. Doudna for helpful comments on the manuscript. Supported by NIH grants GM65050 (J.H.D.C.) and GM072528 (K.F.), National Cancer Institute grant CA92584 (for the SIBYLS and 8.3.1 beamlines), and U.S. Department of Energy grants DE-AC03-76SF00098, KP110201, and LBNL LDRD 366851 (J.H.D.C.) and DE-AC03 76SF00098 (for the SIBYLS and 8.3.1 beamlines).

\section{REFERENCES}

1. Lewis C, Clapp HW. Actinospectacin, a new antibiotic. III. In vitro and in vivo evaluation. Antibiot. Chemother. 1961; 11:127-133.

2. Mason DJ, Dietz A, Smith RM. Actinospectacin, a new antibiotic. I. Discovery and biological properties. Antibiot. Chemother. 1961; 11:118-122.

3. Anderson P. Sensitivity and resistance to spectinomycin in Escherichia coli. J. Bacteriol. 1969; 100:939-947. [PubMed: 16559073]

4. Urassa W, Lyamuya E, Mhalu F. Recent trends on bacterial resistance to antibiotics. East Afr. Med. J. 1997; 74:129-133. [PubMed: 9185405]

5. Brocklehurst P. Antibiotics for gonorrhoea in pregnancy. Cochrane Database Syst. Rev. 2002; 2:CD000098. [PubMed: 12076381]

6. Wisselink HJ, Veldman KT, Van den Eede C, Salmon SA, Mevius DJ. Quantitative susceptibility of Streptococcus sius strains isolated from diseased pigs in seven European countries to antimicrobial agents licensed in veterinary medicine. Vet. Microbiol. 2006; 113:73-82. [PubMed: 16387456]

7. Hoeksema H, Argoudelis AD, Wiley PF. Chemistry of actinospectacin. II. The structure of actinospectacin. J. Am. Chem. Soc. 1962; 84:3212-3213.

8. Bilgin N, Richter AA, Ehrenberg M, Dahlberg AE, Kurland CG. Ribosomal RNA and protein mutants resistant to spectinomycin. EMBO J. 1990; 9:735-739. [PubMed: 2138078]

9. Peske F, Savelsbergh A, Katunin VI, Rodnina MV, Wintermeyer W. Conformational changes of the small ribosomal subunit during elongation factor G-dependent tRNA-mRNA translocation. J. Mol. Biol. 2004; 343:1183-1194. [PubMed: 15491605]

10. Fredrick K, Noller HF. Catalysis of ribosomal translocation by sparsomycin. Science. 2003; 300:1159-1162. [PubMed: 12750524]

11. Valle M, Zavialov A, Sengupta J, Rawat U, Ehrenberg M, Frank J. Locking and unlocking of ribosomal motions. Cell. 2003; 114:123-134. [PubMed: 12859903]

12. Spahn CM, Gomez-Lorenzo MG, Grassucci RA, Jørgensen R, Andersen GR, Beckmann R, Penczek PA, Ballesta JP, Frank J. Domain movements of elongation factor eEF2 and the eukaryotic 80 S ribosome facilitate tRNA translocation. EMBO J. 2004; 23:1008-1019. [PubMed: 14976550]

13. Schuwirth BS, Borovinskaya MA, Hau CW, Zhang W, Vila-Sanjurjo A, Holton JM, Cate JHD. Structures of the bacterial ribosome at $3.5 \AA$ A resolution. Science. 2005; 310:827-834. [PubMed: 16272117] 
14. Savelsbergh A, Katunin VI, Mohr D, Peske F, Rodnina MV, Wintermeyer W. An elongation factor G-induced ribosome rearrangement precedes tRNA-mRNA translocation. Mol. Cell. 2003; 11:1517-1523. [PubMed: 12820965]

15. Horan LH, Noller HF. Intersubunit movement is required for ribosomal translocation. Proc. Natl. Acad. Sci. U.S.A. 2007; 104:4881-4885. [PubMed: 17360328]

16. Frank J, Agrawal RK. A ratchet-like intersubunit reorganization of the ribosome during translocation. Nature. 2000; 406:318-322. [PubMed: 10917535]

17. Spirin AS. Structural dynamic aspects of protein synthesis on ribosomes. Biochimie. 1987; 69:949-956. [PubMed: 3126832]

18. Pan D, Kirillov SV, Cooperman BS. Kinetically competent intermediates in the translocation step of protein synthesis. Mol. Cell. 2007; 25:519-529. [PubMed: 17317625]

19. Moazed D, Noller HF. Intermediate states in the movement of transfer RNA in the ribosome. Nature. 1989; 342:142-148. [PubMed: 2682263]

20. Davies JE, Anderson P, Davies BD. Inhibition of protein synthesis by spectinomycin. Science. 1965; 149:1096-1098. [PubMed: 5318092]

21. Anderson P, Davies JE, Davies BD. Effect of spectinomycin on polypeptide synthesis in extracts of Escherichia coli. J. Mol. Biol. 1967; 29:203-215. [PubMed: 4228335]

22. Moazed D, Noller HF. Interaction of antibiotics with functional sites in 16S ribosomal RNA. Nature. 1987; 327:389-394. [PubMed: 2953976]

23. Fromm H, Edelman M, Aviv D, Galun E. The molecular basis for rRNA-dependent spectinomycin resistance in Nicotiana chloroplasts. EMBO J. 1987; 6:3233-3237. [PubMed: 16453804]

24. Brink MF, Brink G, Verbeet MP, de Boer HA. Spectinomycin interacts specifically with the residues G1064 and C1192 in 16S rRNA, thereby potentially freezing this molecule into an inactive conformation. Nucl. Acids Res. 1994; 22:325-331. [PubMed: 8127669]

25. Noah JW, Dolan MA, Babin P, Wollenzien P. Effects of tetracycline and spectinomycin on the tertiary structure of ribosomal RNA in the Escherichia coli 30 S ribosomal subunit. J. Biol. Chem. 1999; 274:16576-16581. [PubMed: 10347223]

26. Carter AP, Clemons WM, Brodersen DE, Morgan-Warren RJ, Wimberly BT, Ramakrishnan V. Functional insights from the structure of the 30S ribosomal subunit and its interactions with antibiotics. Nature. 2000; 407:340-348. [PubMed: 11014183]

27. Jerinic O, Joseph S. Conformational changes in the ribosome induced by translational miscoding agents. J. Mol. Biol. 2000; 304:707-713. [PubMed: 11124020]

28. Borovinskaya MA, Pai RD, Zhang W, Schuwirth BS, Holton JM, Hirokawa G, Kaji H, Kaji A, Cate JHD. Structural basis for aminoglycoside inhibition of bacterial ribosome recycling. Nat. Struct. Mol. Biol. 2007; 14:727-732. [PubMed: 17660832]

29. Shoji S, Walker SE, Fredrick K. Reverse translocation of tRNA in the ribosome. Mol. Cell. 2006; 24:931-942. [PubMed: 17189194]

30. Konevega AL, Fischer N, Semenkov YP, Stark H, Wintermeyer W, Rodnina MV. Spontaneous reverse movement of mRNA-bound tRNA through the ribosome. Nat. Struct. Mol. Biol. 2007; 14:318-324. [PubMed: 17369838]

31. Taylor DJ, Nilsson J, Merrill AR, Andersen GR, Nissen P, Frank J. Structures of modified eEF2 80 S ribosome complexes reveal the role of GTP hydrolysis in translocation. EMBO J. 2007; 26:2421-2431. [PubMed: 17446867]

32. Takyar S, Hickerson RP, Noller HF. mRNA helicase activity of the ribosome. Cell. 2005; 120:4958. [PubMed: 15652481]

33. Yusupova GZ, Yusupov MM, Cate JHD, Noller HF. The path of messenger RNA through the ribosome. Cell. 2001; 106:233-241. [PubMed: 11511350]

34. Cabañas MJ, Vázquez D, Modolell J. Inhibition of ribosomal translocation by aminoglycoside antibiotics. Biochem. Biophys. Res. Commun. 1978; 83:991-997. [PubMed: 361042]

35. Zierhut G, Piepersberg W, Böck A. Comparative analysis of the effect of aminoglycosides on bacterial protein synthesis in vitro. Eur. J. Biochem. 1979; 98:577-583. [PubMed: 385317]

36. Recht MI, Douthwaite S, Puglisi JD. Basis for prokaryotic specificity of action of aminoglycoside antibiotics. EMBO J. 1999; 18:3133-3138. [PubMed: 10357824] 
37. Otwinowski, Z.; Minor, W. Processing of X-ray diffraction data collected in oscillation mode. In: Sweet, RM.; Carter, CW., Jr., editors. Methods in Enzymology. Academic Press; New York: 1997. p. 307-326.

38. C.C.P.N.4. The CCP4 suite: programs for protein crystallography. Acta Crystallogr. 1994; D50:760-763.

39. Brünger AT, Adams PD, Clore GM, DeLano WL, Gros P, Grosse-Kunstleve RW, Jiang JS, Kuszewski J, Nilges M, Pannu NS. Crystallography \& NMR system: a new software suite for macromolecular structure determination. Acta Crystallogr. 1998; D 54:905-921.

40. Kleywegt GJ, Jones TA. Databases in protein crystallography. Acta Crystallogr. 1998; D 54:1119_ 1131.

41. Cowtan K. General quadratic functions in real and reciprocal space and their application to likelihood phasing. Acta Crystallogr. 2000; D 56:1612-1621.

42. Jones TA, Zou JY, Cowan SW, Kjeldgaard M. Improved methods for building protein models in electron density maps and the location of errors in these models. Acta Crystallogr. 1991; A 47:110-119.

43. Ehrenberg M, Rojas AM, Weiser J, Kurland CG. How many EF-Tu molecules participate in aminoacyl-tRNA binding and peptide bond formation in Escherichia coli translation? J. Mol. Biol. 1990; 211:739-749. [PubMed: 2179565]

44. Hartz D, McPheeters DS, Gold L. Selection of the initiator tRNA by Escherichia coli initiator factors. Genes Dev. 1989; 3:1899-1912. [PubMed: 2695390]

45. Carson, M. Ribbons. In: Sweet, RM.; Carter, CW., Jr., editors. Methods in Enzymology. Academic Press; New York: 1997. p. 493-502.

46. Yusupov MM, Yusupova GZ, Baucom A, Lieberman K, Earnest TN, Cate JHD, Noller HF. Crystal structure of the ribosome at $5.5 \AA$ resolution. Science. 2001; 292:883-896. [PubMed: 11283358]

47. Berk V, Zhang W, Pai RD, Cate JHD. Structural basis for mRNA and tRNA positioning on the ribosome. Proc. Natl. Acad. Sci. U.S.A. 2006; 103:15830-15834. [PubMed: 17038497] 
a
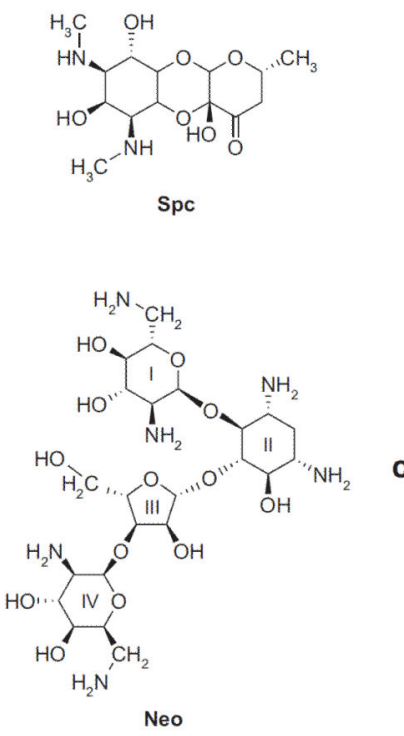

C b
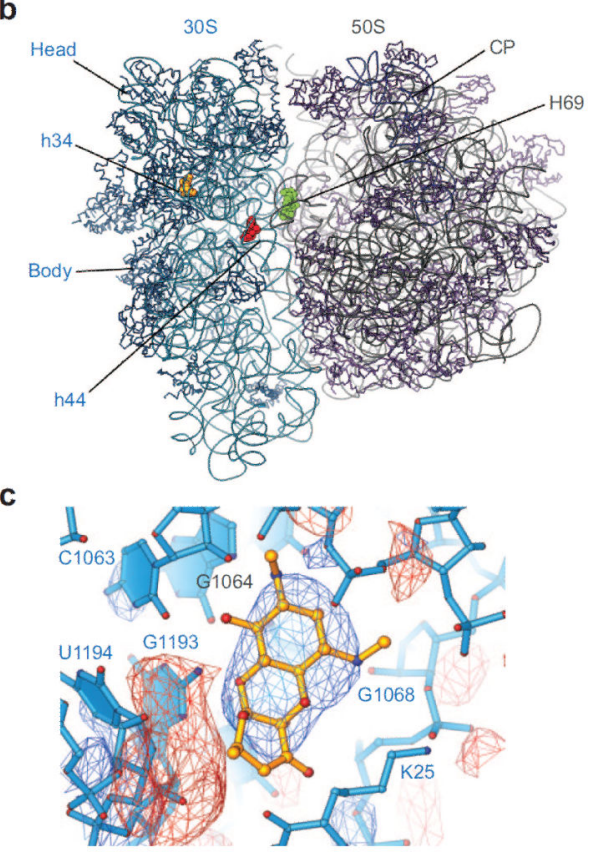

Figure 1.

Structure of spectinomycin and its interactions with the ribosome. a) Chemical structures of antibiotics spectinomycin ( $\mathrm{Spc}$ ) and neomycin (Neo). b) Overview of the binding sites of spectinomycin and neomycin in the context of the 70S ribosome. Spectinomycin bound to h34 is colored gold, and neomycins bound to h44 and H69 are colored red and green, respectively. The $16 \mathrm{~S}$ rRNA, 23S rRNA, and 5S rRNA are shown in light blue, gray, and purple, respectively. Proteins of the small and large subunits are shown in dark blue and magenta, respectively. c) Difference Fourier $\left(F_{\text {obs }}-F_{\text {obs }}\right)$ electron density map of spectinomycin bound to h34 of ribosome II. Observed amplitudes for the unliganded 70S ribosome structure (13) served as a reference. Positive (blue) and negative (red) difference densities are contoured at $3 \mathrm{SD}$ from the mean. 

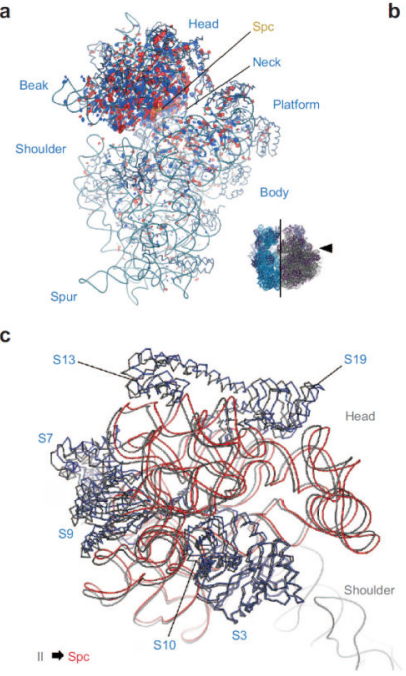

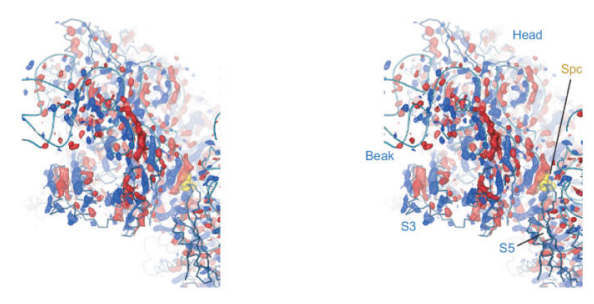

Figure 2.

Conformational changes in the position of the head domain of the 30S subunit induced by spectinomycin. a, b) Difference Fourier $\left(F_{\text {obs }}-F_{\text {obs }}\right)$ electron density map in the context of the 30S ribosomal subunit (panel a) and in the proximity of the spectinomycin binding site in h34 (panel b, stereoview) of ribosome II. Positive (blue) and negative (red) difference densities are contoured at $3 \mathrm{SD}$ from the mean. 16S rRNA is in light blue, and small subunit proteins are in dark blue. Spectinomycin is colored gold. For panel a, the direction of the view is indicated by the ribosome icon. c) Superposition of spectinomycin-bound ( $\mathrm{Spc}$ ) and unliganded (II) (13) conformations of ribosome II in the 70S ribosome crystals. The rRNA is shown in red and gray, and proteins are purple and gray for spectinomycin-bound ( $\mathrm{Spc}$ ) and unliganded (II) (13) conformations of ribo-some II, respectively. d) Superposition of $30 \mathrm{~S}$ subunits within the $70 \mathrm{~S}$ ribosome. Differences in the position of phosphorous atoms in $16 \mathrm{~S}$ rRNA (light colors) and Ca positions in proteins S7, S13, and S19 (darker colors) in the 30S subunit head domain are shown as vectors, as follows. Vectors from apo-ribosome II (13) to spectinomycin-bound ribosome II, red; vectors from spectinomycin-bound ribosome II to spectinomycin-bound ribosome I, blue. The 50S subunit from ribosome I (13) is shown in gray (23S rRNA), purple (5S rRNA), and magenta (large subunit proteins). The direction of the conformational change induced by spectinomycin is indicated by the red arrow. The path tRNAs travel during translocation from the A site to the $\mathrm{E}$ site is indicated by the black arrow. The direction of the view is indicated by the ribosome icon. 
a
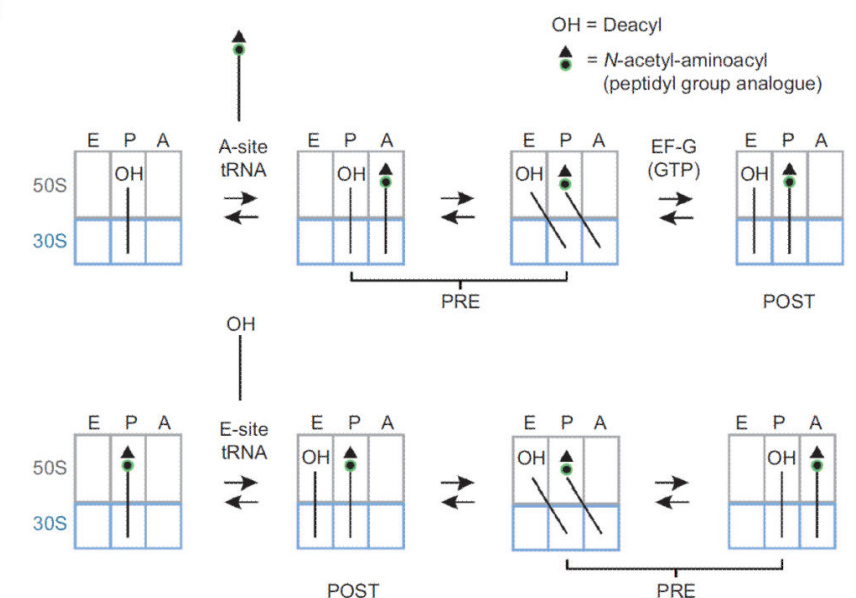

b

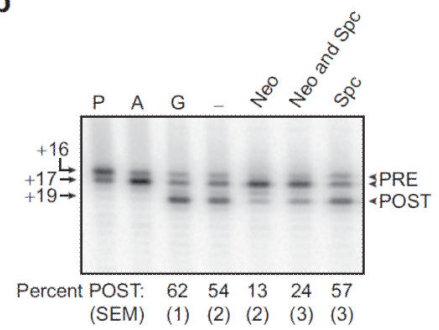

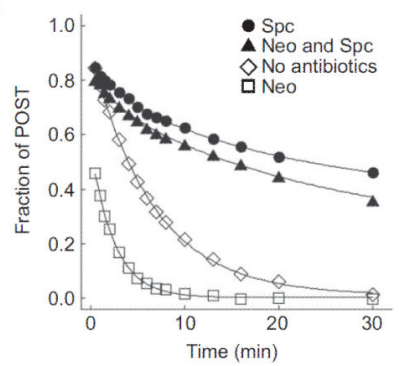

Figure 3.

Effect of spectinomycin on reverse translocation. a) Schematics of the stepwise experimental setup for the EF-G dependent forward translocation (top) and spontaneous reverse translocation (bottom) reactions. The steps and order of antibiotic addition are described in Methods and are not depicted in the figure. b) Reverse translocation promoted by antibiotics in the presence of EF-G and GTP. The P complex corresponds to deacylated tRNA $^{\text {fMet }}$ bound to the P site of the ribosome; the A complex (PRE) forms upon addition of the $N$-acetyl-Val-tRNA ${ }^{\mathrm{Val}}$ to the A site of the P complex; G complex (POST) forms upon addition of EF-G/GTP to the A complex; the other complexes are formed upon subsequent addition of neomycin (Neo), spectinomycin ( $\mathrm{Spc}$ ), both neomycin and spectinomycin (Neo/ $\mathrm{Spc}$ ), or water (-) to the G (POST) complex. The percent of POST complex remaining at the end of the incubation is indicated below each lane. Reported values represent the mean values $( \pm$ SEM) derived from three independent experiments. c) Spontaneous reverse translocation upon addition of E-site tRNA to ribosomes programmed with mRNA and Psite tRNA. Ribosomes were preincubated with spectinomycin prior to E-site tRNA addition in reactions involving spectinomycin (filled symbols). In reactions involving neomycin, neomycin was added simultaneously with E-site tRNA. Each experiment was carried out in triplicate. Kinetic parameters derived from the fitting of the data are reported in Table 1. 


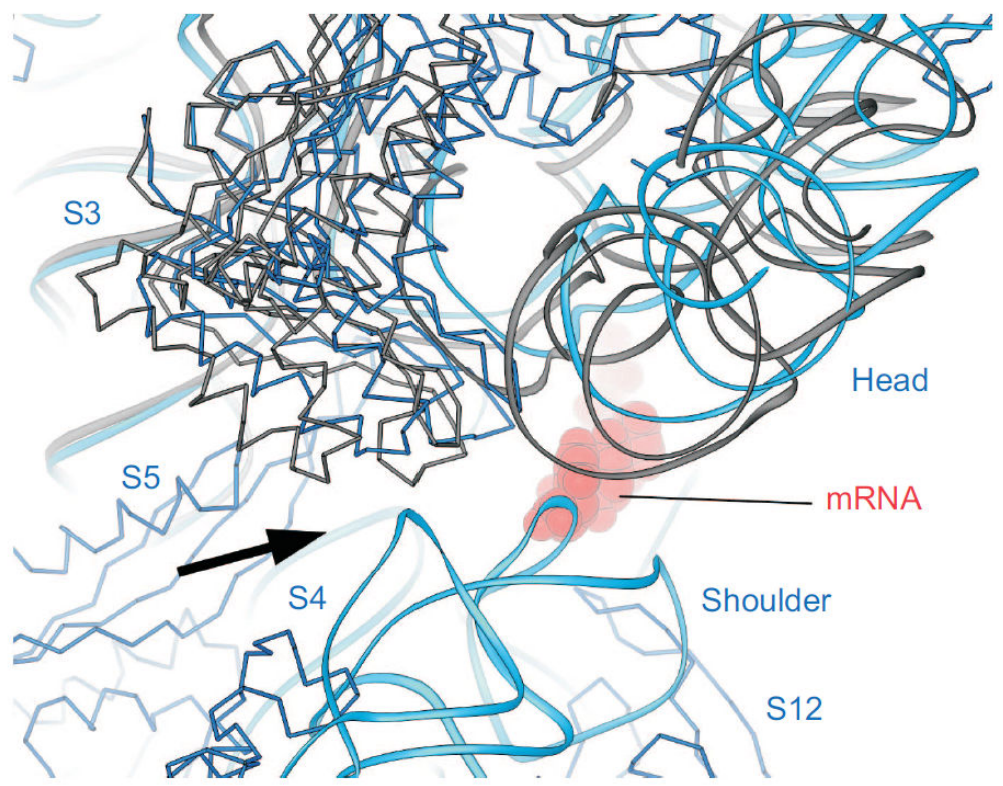

Figure 4.

Conformational changes in the mRNA downstream tunnel that accompany mRNA unwinding and movement through the ribosome, viewed from the solvent side of the $30 \mathrm{~S}$ subunit. The mRNA from ref 46 superimposed with the ribosome structures $(13,47)$ is shown in red. The 16S rRNA is shown in light blue, and proteins S3, S4, and S5 are dark blue for the conformation of the ribosome that precedes mRNA and tRNA movement on the small subunit, with a fully rotated $30 \mathrm{~S}$ head, i.e., unliganded ribosome II (13). For the pretranslocation-like conformation of the ribosome, with a 30S subunit head not rotated, taken from a structure of the ribosome in complex with mRNA and a P-site tRNA mimic (47), the head domain of the 30S subunit, which adopts a different conformation compared to that in unliganded ribosome II, is shown in gray. The body of the 30S subunit in the pretranslocation-like state (not shown) is essentially identical in conformation to that in unliganded ribosome II $(13,47)$. The location of the mRNA downstream tunnel is indicated by the arrow. 


\section{TABLE 1}

Effect of spectinomycin and neomycin on the rate of spontaneous reverse translocation

\begin{tabular}{lllll}
\hline Antibiotic & $\boldsymbol{k}_{\mathbf{o b s} \mathbf{1}}\left(\mathbf{m i n}^{-\mathbf{1}}\right)$ & $\boldsymbol{A}_{\mathbf{1}} \boldsymbol{a}$ & $\boldsymbol{k}_{\mathbf{o b s 2}}\left(\mathbf{m i n}^{-\mathbf{1}}\right)$ & $\boldsymbol{A}_{\mathbf{2}} \boldsymbol{a}$ \\
\hline Water & $0.15 \pm 0.01$ & $0.98 \pm 0.01$ & $\mathrm{n} / \mathrm{a}$ & $\mathrm{n} / \mathrm{a}$ \\
Neomycin & $0.39 \pm 0.02$ & $0.99 \pm 0.01$ & $\mathrm{n} / \mathrm{a}$ & $\mathrm{n} / \mathrm{a}$ \\
Spectinomycin & $0.05 \pm 0.01$ & $0.29 \pm 0.02$ & $0.54 \pm 0.02$ & $0.26 \pm 0.02$ \\
Spc + Neo & $0.07 \pm 0.02$ & $0.33 \pm 0.03$ & $0.72 \pm 0.01$ & $0.43 \pm 0.02$ \\
& \multicolumn{5}{c}{ Preincubated with spectinomycin } \\
Spectinomycin & $0.04 \pm 0.01$ & $0.54 \pm 0.06$ & $0.30 \pm 0.03$ & $0.13 \pm 0.01$ \\
Spc + Neo & $0.06 \pm 0.01$ & $0.56 \pm 0.01$ & $0.44 \pm 0.02$ & $0.06 \pm 0.05$ \\
\hline
\end{tabular}

$a_{A_{1}}$ and $A_{2}$ are amplitudes of the reactions (Methods, Supporting Information). 
TABLE 2

Diffraction and refinement statistics for $70 \mathrm{~S}$ ribosome in complexes with spectinomycin and neomycin ${ }^{a}$

\begin{tabular}{lll}
\hline Dataset & + Spectinomycin & + Spectinomycin + Neomycin \\
\hline Resolution $(\AA)$ & $183-3.93(183-4.2)^{b}$ & $182-3.5(182-3.85)^{b}$ \\
Completeness (\%) & $75.8(89.4)^{b}$ & $72.1(91.3)^{b}$ \\
No. of reflections & 390,966 & 521,699 \\
No. of crystals & 7 & 6 \\
Redundancy & 3.4 & 3.3 \\
$I / \sigma$ & 8.4 & 8.6 \\
Overall $\chi^{2}$ & 1.4 & 1.4 \\
$R_{\text {merge }}(\%)$ & 10.9 & 10.4 \\
& & \\
Resolution $(\AA)$ & $70-3.93$ & $70-3.5$ \\
No. of reflections & 390,759 & 521,145 \\
$R_{\text {free Set }}$ & 19,244 & 25,274 \\
No. of atoms & 284,067 & 284,235 \\
$R / R_{\text {free }}(\%)$ & $25.8 / 31.1$ & $26.4 / 30.6$ \\
\hline
\end{tabular}

${ }^{a}$ Space group $P 2{ }_{12}{ }_{12} 1$. Unit cell $a=208.9 \AA, b=379.2 \AA, c=739.3 \AA, \alpha=\beta=\gamma=90^{\circ}$.

${ }^{b}$ Values in parenthesis are for the resolution range with a high level of data completion. 